\title{
Pembrolizumab monotherapy or combination therapy for bone metastases in advanced non-small cell lung cancer: a real-world retrospective study
}

\author{
Huiping Qiang ${ }^{1 \#}$, Yuqiong Lei", Yinchen Shen ${ }^{1}$, Jiaqi Li ${ }^{1}$, Hua Zhong ${ }^{1}$, Runbo Zhong', Xueyan Zhang ${ }^{1}$, \\ Qing Chang ${ }^{1}$, Jiahuan Lu ${ }^{1}$, Hui Feng ${ }^{1}$, Yan Zhu ${ }^{1}$, Alfredo Addeo ${ }^{3}$, Giuseppe L. Banna ${ }^{4}$, In-Jae Oh ${ }^{5}$, \\ Jialin Qian $^{1 \#}$, Tianqing Chu ${ }^{1 \#}$ \\ ${ }^{1}$ Department of Respiratory Medicine, Shanghai Chest Hospital, Shanghai Jiao Tong University, Shanghai, China; ${ }^{2}$ Department of Respiratory \\ Medicine, Shanghai Sixth People's Hospital, Shanghai Jiao Tong University, Shanghai, China; ${ }^{3}$ Oncology Department, University Hospital of \\ Geneva, Geneva, Switzerland; ${ }^{4}$ Candiolo Cancer Institute, FPO-IRCCS, Candiolo, Turin, Italy; ${ }^{5}$ Department of Internal Medicine, Chonnam \\ National University Medical School and Hwasun Hospital, Jeonnam, Republic of Korea \\ Contributions: (I) Conception and design: J Qian, T Chu; (II) Administrative support: None; (III) Provision of study materials or patients: Y Shen, \\ H Zhong, R Zhong, X Zhang, J Qian, T Chu; (IV) Collection and assembly of data: J Li, Q Chang, J Lu, H Feng, Y Zhu; (V) Data analysis and \\ interpretation: H Qiang, Y Lei; (VI) Manuscript writing: All authors; (VII) Final approval of manuscript: All authors. \\ \#These authors contributed equally to this work. \\ Correspondence to: Jialin Qian; Tianqing Chu. Department of Respiratory Medicine, Shanghai Chest Hospital, Shanghai Jiao Tong University, No. \\ 241, Huaihai West Road, Shanghai 200030, China. Email: jialin_qian@aliyun.com; tianqing_chu@126.com.
}

Background: The incidence of bone metastases in non-small cell lung cancer (NSCLC) patients is about $30-40 \%$ and bone-related events can seriously affect quality of life. Immune checkpoint inhibitor (ICI) therapy has become the standard treatment for advanced NSCLC patients. However, the specific efficacy of ICIs in NSCLC patients with bone metastases remains unclear. The aim of the present study was to explore the prognosis of immunotherapy in this population and to find potential biomarkers.

Methods: In this retrospective study, a total of 110 advanced NSCLC patients with bone metastases who received pembrolizumab therapy were enrolled. Patient characteristics; palliative bone radiotherapy or bone-targeted therapy; serum levels of lactate dehydrogenase (LDH), and the neutrophil-to-lymphocyte ratio (NLR) at baseline were assessed. The correlation of these factors with progression-free survival (PFS), overall survival (OS), and objective response rate (ORR) was analyzed.

Results: The ORR of the total population was 29.1\%, and PFS and OS were 7.0 and 14.8 months, respectively. Fifty-eight patients $(52.7 \%)$ received pembrolizumab treatment as first-line therapy, and 52 patients (47.3\%) as second-line therapy or beyond [ORR: 41.4\% vs. 15.4\%, $\mathrm{P}=0.011$; PFS: 9.0 vs. 4.0 months, $\mathrm{P}=0.004$; OS: not reached (NR) vs. 11.5 months, $\mathrm{P}<0.0001]$. Bone therapy, including palliative bone radiotherapy and bone-targeted therapy, increased the ORR (34.9\% vs. $11.1 \%, \mathrm{P}<0.0001)$ and prolonged PFS (8.5 vs. 2.0 months, $\mathrm{P}=0.002)$. Eastern Cooperative Oncology Group performance status score of $0-1$ [OS: hazard ratio $(\mathrm{HR})=0.117, \mathrm{P}<0.0001]$ and first-line pembrolizumab therapy $(\mathrm{OS}: \mathrm{HR}=0.372, \mathrm{P}=0.004)$ were independent predictors of OS. Patients whose baseline serum LDH level was $\leq 240.5$ IU/L (NR $v$ s. 10.0 months, $\mathrm{P}<0.0001$ ) or $\mathrm{NLR} \leq 5.55$ (NR vs. 18.0 months, $\mathrm{P}=0.039$ ) showed longer $\mathrm{OS}$.

Conclusions: The efficacy of Pembrolizumab therapy is confirmed in advanced NSCLC patients with bone metastases, particularly when palliative bone radiotherapy or bone-targeted therapy is delivered. Baseline serum LDH level $\leq 240.5$ IU/L and NLR $\leq 5.55$ might predict the prognosis of patients with bone metastases from advanced NSCLC treated with immunotherapy.

Keywords: Non-small cell lung cancer (NSCLC); immune checkpoint inhibitors (ICIs); bone metastases; palliative radiotherapy; bone-targeted therapy 
Submitted Oct 26, 2021. Accepted for publication Jan 19, 2022.

doi: $10.21037 /$ tlcr-21-1033

View this article at: https://dx.doi.org/10.21037/tlcr-21-1033

\section{Introduction}

Lung cancer is the leading cause of cancer-related deaths globally. In 2020, approximately 1.8 million patients died of lung cancer worldwide (18\% of all cancer-related deaths) (1). Non-small cell lung cancer (NSCLC) accounts for more than $80 \%$ of all lung cancers. In recent years, immune checkpoint inhibitor (ICI) therapy has become one of the main treatment strategies for advanced or metastatic NSCLC without driver gene mutations. The most used ICIs target the programmed death receptor-1/ programmed death ligand-1 (PD-1/PD-L1) pathway, including nivolumab, pembrolizumab, and atezolizumab. Pembrolizumab is a highly selective humanized monoclonal antibody responsible for inhibiting the PD-1 receptor on $\mathrm{T}$ cells while preserving the antitumor function of host immune cells. Several preclinical and clinical studies have shown that its addition to tumor management led to a regression of tumor size in NSCLC patients. Pembrolizumab monotherapy for PD-L1 positive NSCLC or combined with chemotherapy has become the first-line treatment for advanced NSCLC $(2,3)$.

Most lung cancer patients are diagnosed with locally advanced or metastatic lung cancer (4). The bone is one of the most common metastatic sites of lung cancer (5). About $30-40 \%$ of advanced NSCLC patients will develop bone metastases, which can cause severe pain and fractures, leading to hospitalizations, loss of autonomy and poor quality of life (6). Treatment strategies for bone metastases include systemic treatment of the primary tumor, combined with palliative radiotherapy and bone-targeted therapy $(7,8)$. Combination of denosumab and ICIs, including pembrolizumab, has been clarified to be effective for bone metastases in lung cancer (9). However, real-world data on the impact of metastatic lesions and treatment regimens on immunotherapy is lacking. As reported, numerous routine blood parameters have been investigated as inflammatory biomarkers in patients with cancer, such as absolute neutrophil count, absolute platelet count and serum levels of lactate dehydrogenase (LDH), which are also associated with poor outcomes (10). The neutrophil-to-lymphocyte ratio (NLR) is a novel predictor of inflammatory status in NSCLC and may represents the frequency and activity of myeloid-derived suppressor cells (MDSCs) (11). The two indicators are simple and easy to access from blood routine examination. Therefore, we conducted this retrospective clinical study to evaluate the efficacy of immunotherapy in these patients and explored potential biomarkers. We present the following article in accordance with the STROBE reporting checklist (available at https://tlcr. amegroups.com/article/view/10.21037/tlcr-21-1033/rc).

\section{Methods}

\section{Patient characteristics}

Patients who were diagnosed with advanced NSCLC with bone metastases between July 2017 and July 2020, and received pembrolizumab therapy at Shanghai Chest Hospital Affiliated to Shanghai Jiao Tong University, were enrolled in this retrospective study. Patient characteristics were collected, including sex, age, Eastern Cooperative Oncology Group performance status (ECOG PS), smoking history, histological subtype, epidermal growth factor receptor (EGFR) mutation status, PD-L1 expression, treatment strategy (i.e., monotherapy or combination), treatment line, sites and number of bone metastases, other distant metastatic sites, baseline neutrophil and lymphocyte count, and serum levels of LDH. Blood information were collected from reports routine blood samples performed within seven days prior to treatment initiation and analyzed by the local laboratory. All procedures performed in this study involving human participants were in accordance with the Declaration of Helsinki (as revised in 2013). The study was approved by the Ethics Committee of Shanghai Chest Hospital Affiliated to Shanghai Jiao Tong University (No. IS21110) and informed consent was taken from all the patients.

\section{Treatment strategies}

For advanced NSCLC patients without sensitizing EGFR or ALK mutations, they could receive first-line pembrolizumab monotherapy with PD-L1 positive or combination therapy regardless of PD-L1 expression. The remaining patients who have been treated previously could receive pembrolizumab monotherapy or combination therapy as neoadjuvant therapy or second-line and above treatment. All patients received at least 2 cycles 
of pembrolizumab as monotherapy or in combination with platinum-based chemotherapy or antiangiogenetic drugs at a dose of $200 \mathrm{mg}$ per intravenous infusion every 3 weeks until disease progression or unacceptable toxicity occurred. Palliative bone radiotherapy, including single and multiple fractional radiotherapy, was offered if feasible aiming at symptom control. Bone-targeted therapy with bisphosphonate administration, like pamidronate disodium and zoledronate sodium, was intravenously injected every pembrolizumab cycle continuously.

\section{Efficacy evaluation}

Immunotherapy efficacy was evaluated according to the Response Evaluation Criteria In Solid Tumors (version 1.1), which defines complete response (CR), partial response (PR), stable disease (SD) and progressive disease (PD) (12). Objective response rate (ORR) was calculated as the percentage of patients with evaluated CR or PR. Progression-free survival (PFS) was calculated from the first pembrolizumab administration to the disease progression by physician assessment or death or last follow up (February 1, 2021). Overall survival (OS) was estimated from the pembrolizumab start date until death from any cause or last follow up. To detect the sites and number of bone metastases, patients were assessed by bone scintigraphy, 18-fluorodeoxyglucose positron emission tomography/ computed tomography, or magnetic resonance imaging before pembrolizumab treatment start.

\section{Statistical analysis}

Statistical analysis was performed using SPSS version 26.0 (IBM, Armonk, NY, USA). Baseline clinical characteristics were listed by numbers and percentages. The $\chi^{2}$-test was used to measure the association between patient characteristics and ORR. The Kaplan-Meier method was used to analyze the survival probability, and log-rank test to calculate the significance of differences. Cox proportional hazard model was applied for the univariate and multivariate analyses to calculate the hazard ratios (HRs) and 95\% confidence intervals on survival outcomes. Pearson's correlation test was used to investigate association between serum biomarkers and survival. To evaluate values of serum levels of these biomarkers in the prediction of patient survival, receiveroperating characteristic (ROC) curve analysis was performed. $P$ values in the study were 2 sided and considered statistically significant when less than 0.05 .

\section{Results}

\section{Patient characteristics}

The clinical characteristics of all patients are shown in Table 1. One-hundred-ten advanced NSCLC patients with bone metastases were identified; $76.4 \%$ were male (84/110) and 23.6\% were female (26/110). Among them, $94.5 \%(104 / 110)$ had an ECOG PS score of $0-1,60 \%$ $(66 / 110)$ a smoking history, and $56.3 \%(62 / 110)$ were diagnosed with lung adenocarcinoma. The proportion of patients who had EGFR mutation positive tumors was $18.2 \%(20 / 110)$. PD-L1 expression was detected in 59 patients $(53.6 \%)$ before immunotherapy, of which $55.9 \%(33 / 59)$ were PD-L1 expression positive by $22 \mathrm{C} 3$ pharmDx immunohistochemistry assay. In addition to bone metastases, 74 patients $(67.3 \%)$ had other sites of distant metastases. Among this population, the baseline incidence of brain metastases was $23.0 \%$ (17/74), liver metastases $23.0 \%$ (17/74), adrenal metastases $9.5 \%$ (7/74), and pleural metastases $35.1 \%$ (26/74). Thirty-eight patients (34.5\%) were treated with pembrolizumab monotherapy while 72 $(65.5 \%)$ with combination therapy. Fifty-eight patients $(52.7 \%)$ received pembrolizumab as first-line treatment, the remaining 52 patients $(47.3 \%)$ as second-line or beyond therapy. In terms of efficacy, $29.1 \%(32 / 110)$ and $44.5 \%$ (49/110) patients achieved PR and SD respectively, and $26.4 \%(29 / 110)$ developed disease progression.

\section{Sites of bone metastases and number of lesions}

Sites of bone metastases and the number of lesions for all patients are shown in Figure 1. The most common site of bone metastases was the spine (50.9\%), followed by ribs (41.8\%) and pelvis $(37.3 \%)$. Fifty patients $(45.5 \%)$ had one, while the other 60 patients $(60 / 100,54.4 \%)$ had $\geq 2$ bone lesions.

\section{Efficacy and prognosis of patients with bone metastases treated with pembrolizumab}

As shown in Figure 2, in the overall patient cohort, the ORR was $29.1 \%$, and the median PFS and OS were 7.0 and 14.8 months, respectively. Compared with second-line or beyond therapy, patients receiving first-line pembrolizumab had higher ORR (41.4\% vs. $15.4 \%, \mathrm{P}=0.011)$, longer PFS (9.0 vs. 4.0 months, $\mathrm{P}=0.004)$ and $\mathrm{OS}$ [not reached (NR) vs. 11.5 months, $\mathrm{P}<0.0001$ ] (Figure 3). $\mathrm{PD}-\mathrm{L} 1$ expression was detected in 59 patients (53.6\%) among all enrolled in our study. Among those whose PD-L1 expression 
Table 1 Patient characteristics

\begin{tabular}{|c|c|}
\hline Characteristic & $\mathrm{n}=110(\%)$ \\
\hline \multicolumn{2}{|l|}{ Sex } \\
\hline Male & $84(76.4)$ \\
\hline Female & $26(23.6)$ \\
\hline \multicolumn{2}{|l|}{ Age (years) } \\
\hline$<65$ & $56(50.9)$ \\
\hline$\geq 65$ & $54(49.1)$ \\
\hline \multicolumn{2}{|l|}{ ECOG PS } \\
\hline $0-1$ & $104(94.5)$ \\
\hline$\geq 2$ & $6(5.5)$ \\
\hline \multicolumn{2}{|l|}{ Smoking history } \\
\hline Yes & $66(60.0)$ \\
\hline No & $44(40.0)$ \\
\hline \multicolumn{2}{|l|}{ Histological subtype } \\
\hline Adenocarcinoma & $62(56.3)$ \\
\hline Squamous carcinoma & $30(27.3)$ \\
\hline NSCLC NOS & $18(16.4)$ \\
\hline \multicolumn{2}{|l|}{ EGFR status } \\
\hline Positive & $20(18.2)$ \\
\hline Negative & $90(81.8)$ \\
\hline \multicolumn{2}{|l|}{ PD-L1 expression } \\
\hline$\leq 1 \%$ & $26(23.6)$ \\
\hline $1-49 \%$ & $12(10.9)$ \\
\hline$\geq 50 \%$ & $21(19.1)$ \\
\hline Unknown & $51(46.4)$ \\
\hline \multicolumn{2}{|l|}{ Other distant metastases } \\
\hline Yes & $74(67.3)$ \\
\hline No & $36(32.7)$ \\
\hline \multicolumn{2}{|l|}{ Brain metastasis } \\
\hline Yes & $17(23.0)$ \\
\hline No & $57(77.0)$ \\
\hline \multicolumn{2}{|l|}{ Liver metastasis } \\
\hline Yes & $17(23.0)$ \\
\hline No & $57(77.0)$ \\
\hline \multicolumn{2}{|l|}{ Adrenal metastasis } \\
\hline Yes & $7(9.5)$ \\
\hline No & $67(90.5)$ \\
\hline
\end{tabular}

Table 1 (continued)
Table 1 (continued)

\begin{tabular}{ll}
\hline Characteristic & $\mathrm{n}=110(\%)$ \\
\hline Pleural metastasis & $26(35.1)$ \\
Yes & $48(64.9)$ \\
No & \\
Treatment strategy & $38(34.5)$ \\
Pembrolizumab monotherapy & $72(65.5)$ \\
Pembrolizumab combination therapy & \\
Treatment line & $58(52.7)$ \\
1 & $52(47.3)$ \\
$\geq 2$
\end{tabular}

ECOG PS, Eastern Cooperation Oncology Group performance status; EGFR, epidermal growth factor receptor; NSCLC, nonsmall cell lung cancer; PD-L1, programmed cell death-ligand 1.
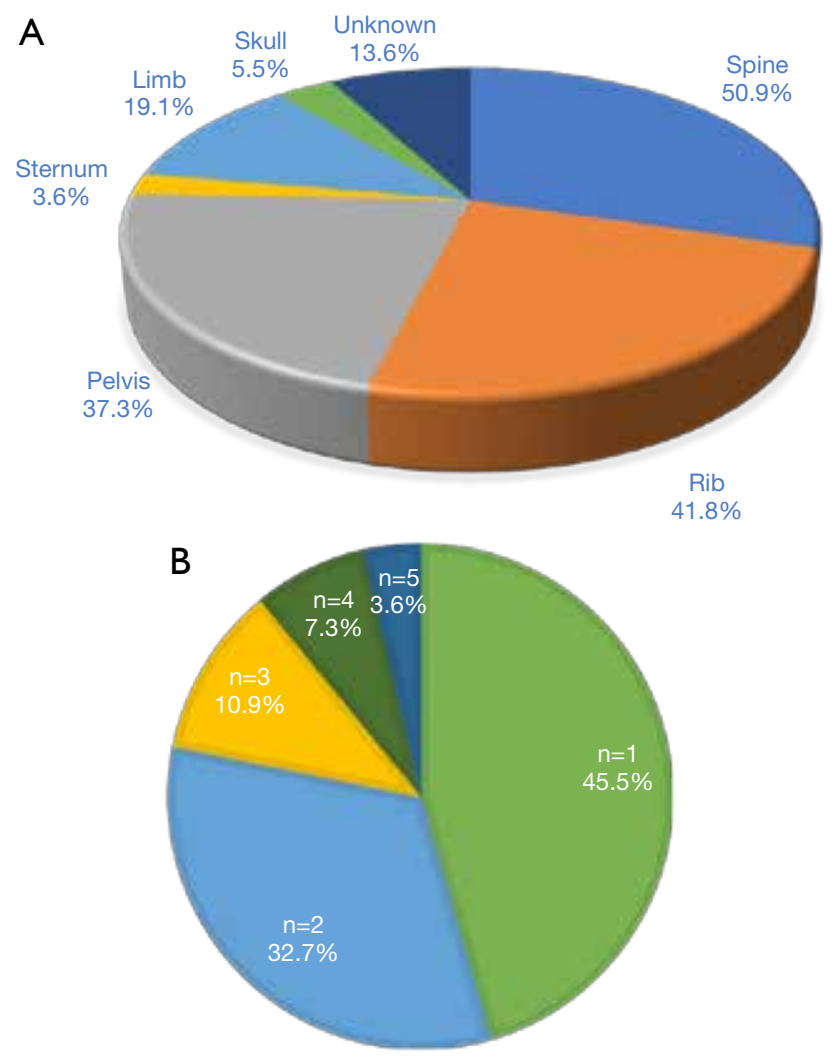

Figure 1 Sites (A) and number of bone metastases (B) in all patients $(n=110)$. 

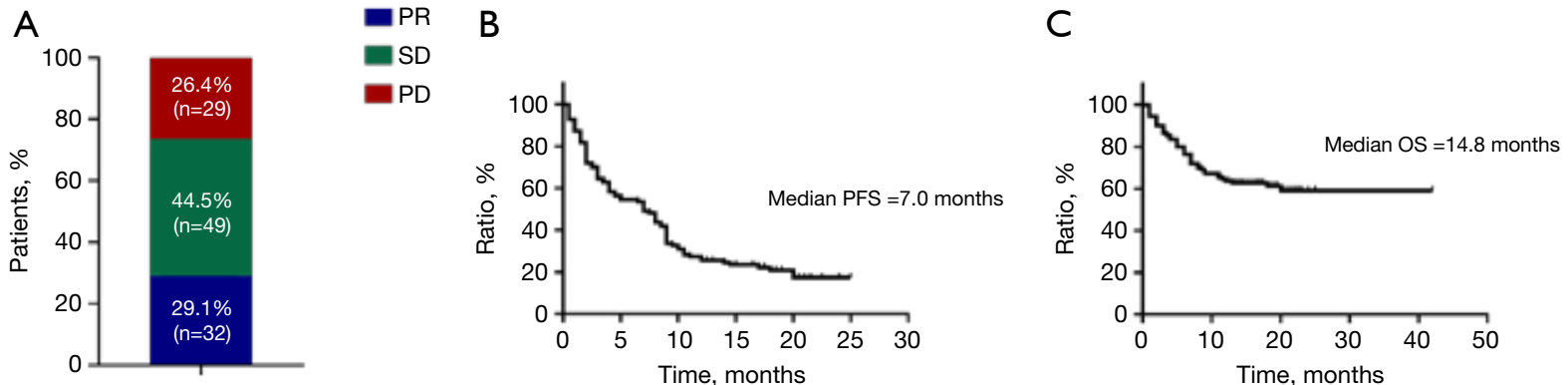

Figure 2 Efficacy and survival characteristics of all patients. (A) Objective response rate, (B) progression-free survival, and (C) overall survival for all patients $(\mathrm{n}=110)$. $\mathrm{PD}$, progressive disease; $\mathrm{SD}$, stable disease; $\mathrm{PR}$, partial response.
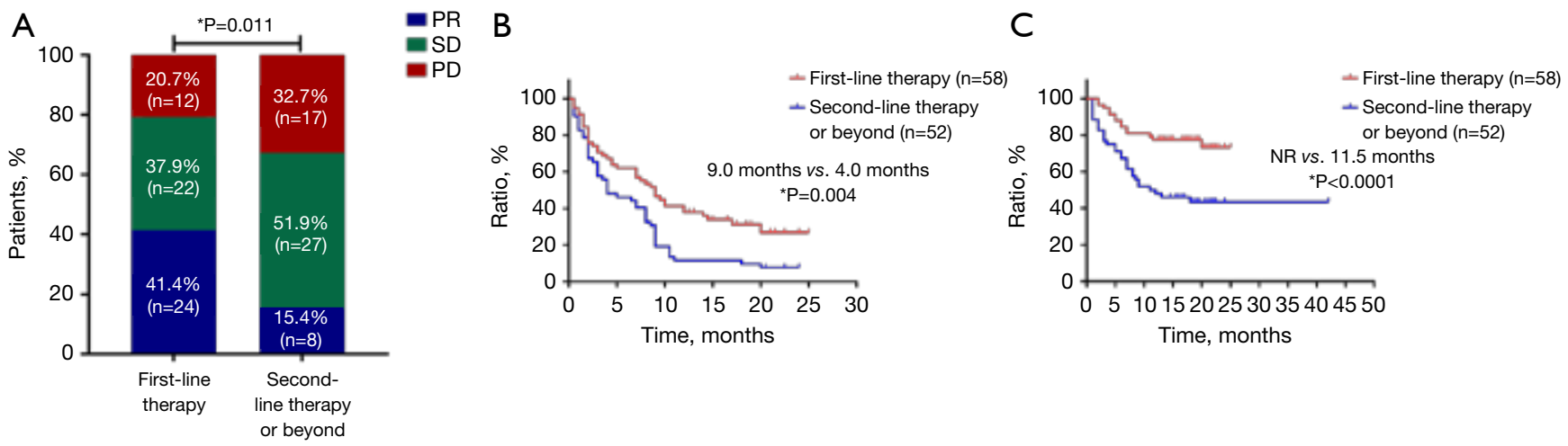

Figure 3 Efficacy and survival characteristics of patients with different immunotherapy lines. (A) Objective response rate, (B) progressionfree survival, and $(\mathrm{C})$ overall survival in patients with different pembrolizumab treatment lines $(\mathrm{n}=110)$. * $\mathrm{P}<0.05$. PD, progressive disease; $\mathrm{SD}$, stable disease; NR, not reached; PR, partial response.
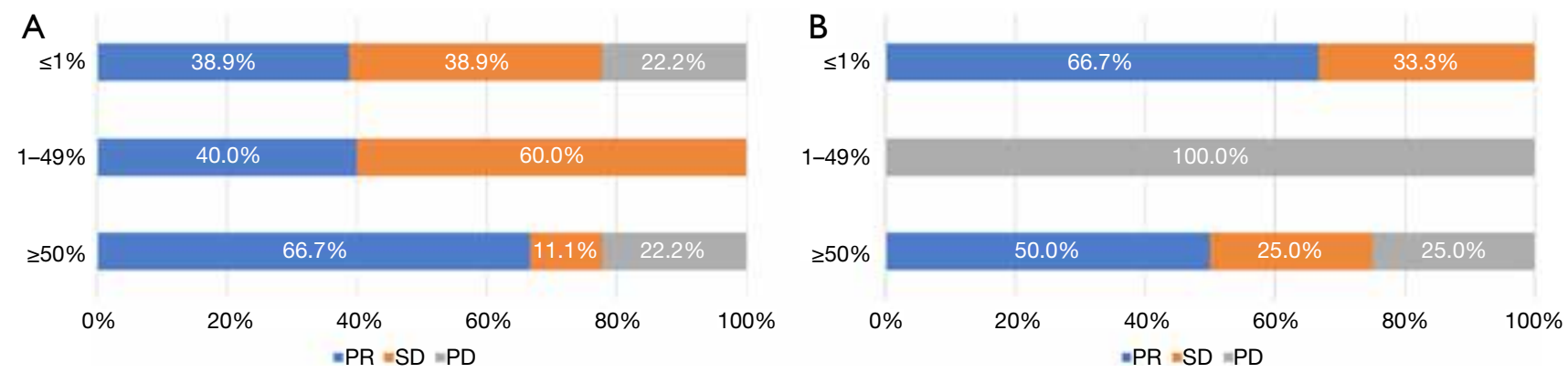

Figure 4 Ratios of programmed cell death-ligand 1 expression to efficacy in patients receiving first-line pembrolizumab combination therapy ( $\mathrm{n}=47)(\mathrm{A})$ and first-line pembrolizumab monotherapy $(\mathrm{n}=11)(\mathrm{B}) . \mathrm{PD}$, progressive disease; SD; stable disease; PR, partial response.

$\geq 50 \%(21 / 59,19.1 \%)$, the proportion of achieving PR after first-line pembrolizumab combination therapy $(6 / 9$, $66.7 \%)$ was higher than that of monotherapy (2/4, $50 \%$, $\mathrm{P}<0.0001)$. Interestingly, for low and negative PD-L1 patients receiving pembrolizumab combination therapy regardless of treatment line, the proportion of achieving
PR (11/29, 37.9\%) was also significantly higher than that of monotherapy $(2 / 9,22.2 \%, \mathrm{P}<0.0001)$ (Figure 4).

The OS of patients with only one bone lesion was significantly longer than those with $\geq 2$ lesions (PFS: 9.0 vs. 4.8 months, $\mathrm{P}=0.070$; OS: NR vs. $\mathrm{NR}$ months, $\mathrm{P}=0.045)$ (Figure 5). 

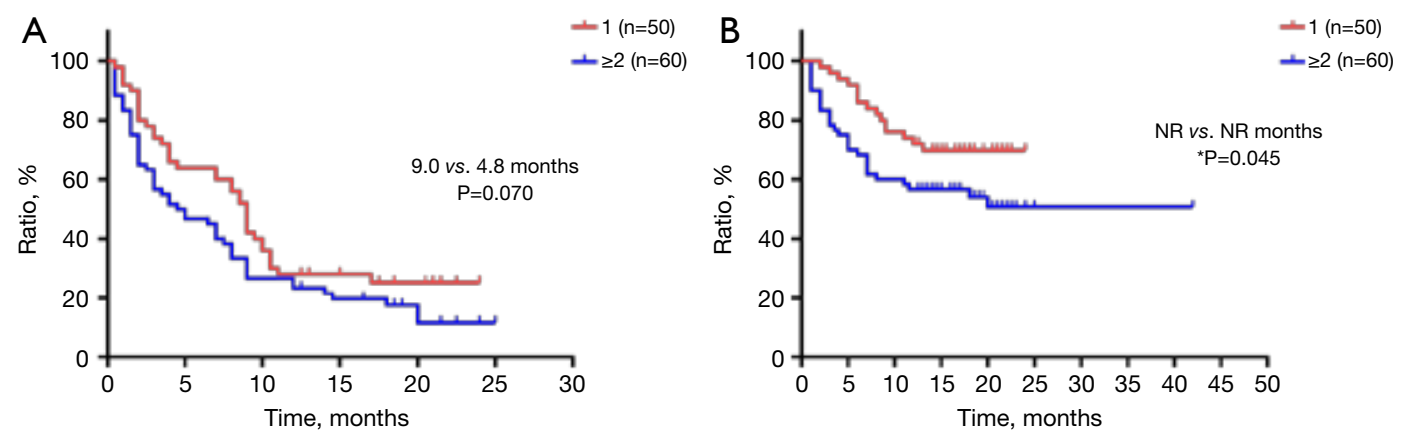

Figure 5 Survival characteristics of patients with different numbers of bone metastases. (A) Progression-free survival and (B) overall survival in patients with different numbers of bone metastases. *, $\mathrm{P}<0.05$. NR, not reached.
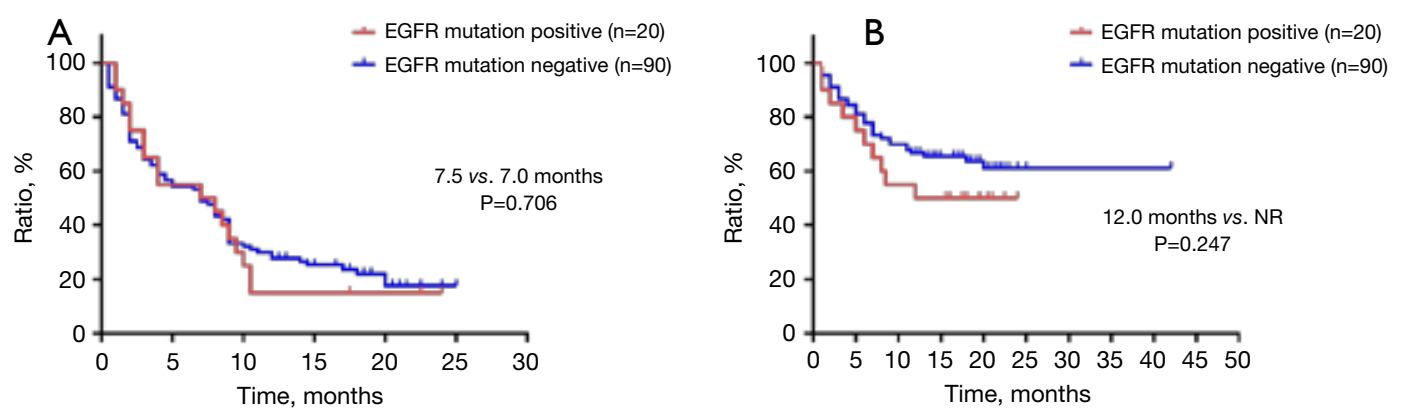

Figure 6 Survival characteristics of patients with different EGFR mutation statuses. (A) Progression-free survival and (B) overall survival in patients with different EGFR mutation statuses. EGFR, epidermal growth factor receptor; NR, not reached.

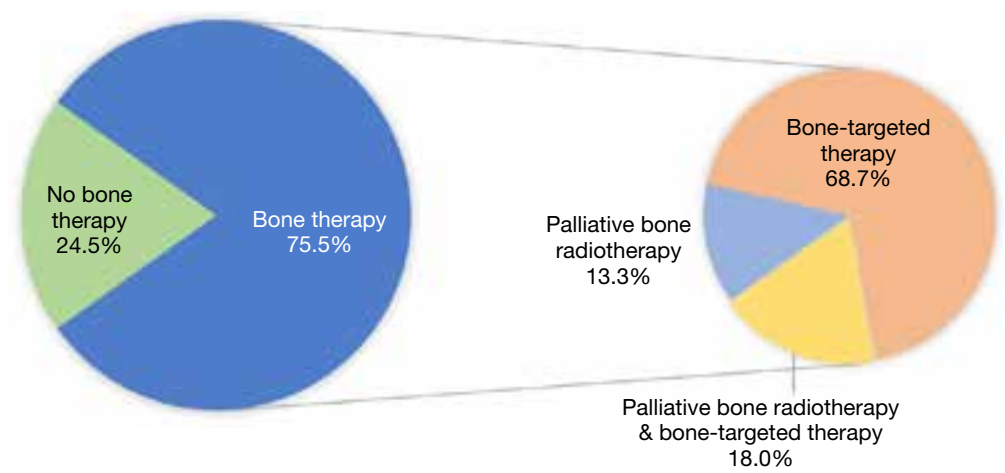

Figure 7 Condition of patients receiving bone therapy $(\mathrm{n}=110)$.

No statistical significant difference was observed between patients with EGFR mutation negative and positive tumors (PFS: 7.5 vs. 7.0 months, $\mathrm{P}=0.706$; OS: NR vs. 12.0 months, $\mathrm{P}=0.247$ ) (Figure 6).

\section{Efficacy of bone therapy and patient prognosis}

Of the 110 patients, $83(75.5 \%)$ received bone therapy.
Among them, 15 patients (18\%) received both palliative bone radiotherapy and bone-targeted therapy, and the remaining $57(68.7 \%)$ and 11 patients $(13.3 \%)$ received only palliative bone radiotherapy or bone-targeted therapy, respectively (Figure 7). ORR was significantly increased (34.9\% vs. $11.1 \%, \mathrm{P}<0.000)$ and $\mathrm{PFS}$ was significantly prolonged ( 8.5 vs. 2.0 months, $\mathrm{P}=0.002)$ in patients who received either palliative bone radiotherapy or bone- 

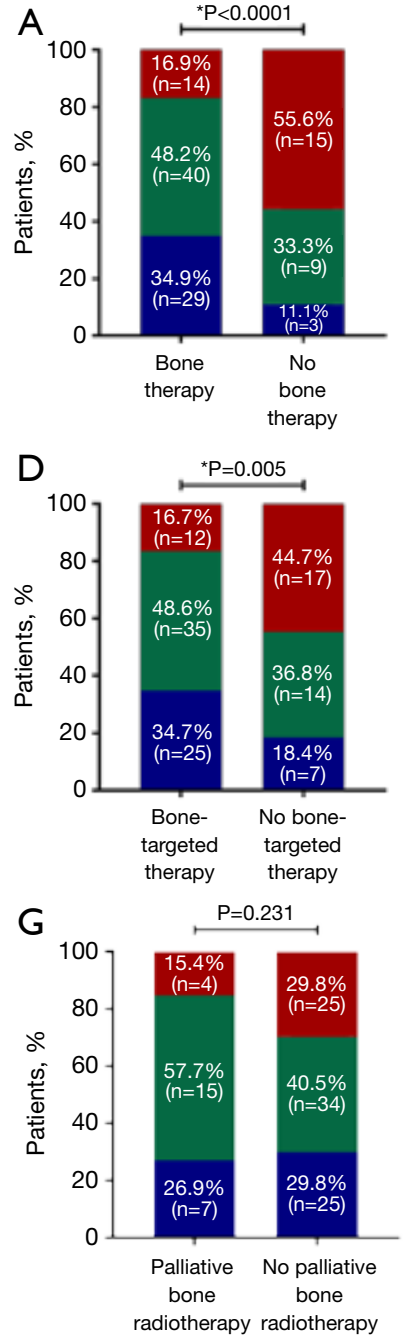

$B$

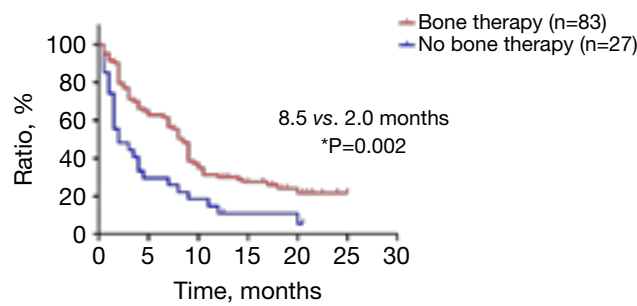

$\mathrm{E}$

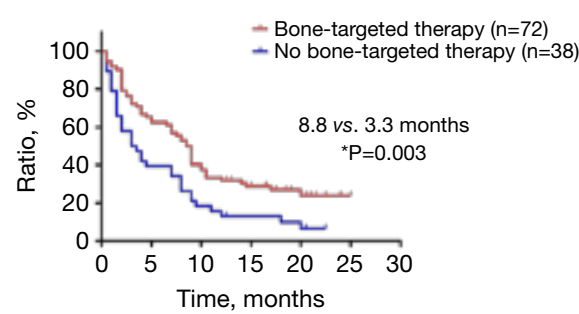

$=\operatorname{PR} H$

$=\mathrm{PD}$

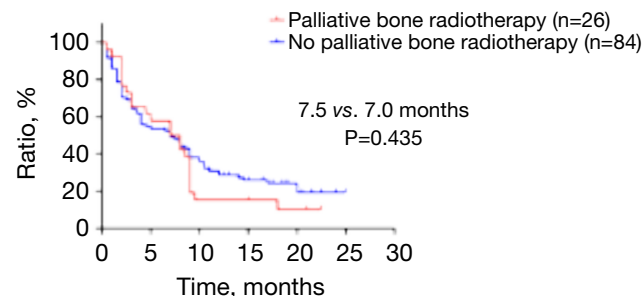

C

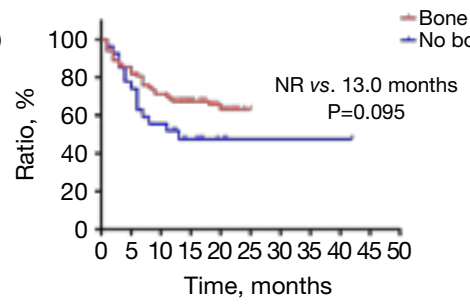

$\mathrm{F}$

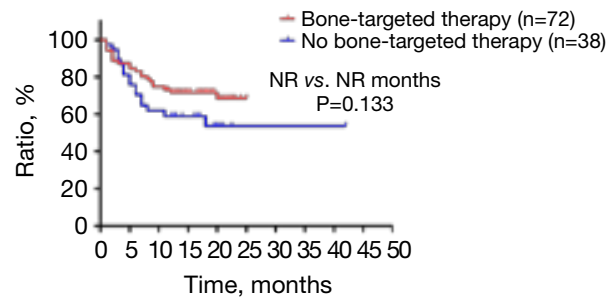

I

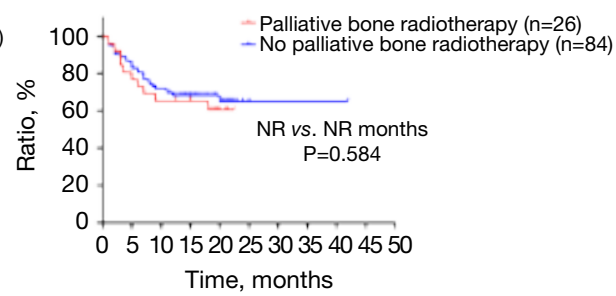

Figure 8 Efficacy and survival characteristics of patients with different bone therapies. (A) ORR, (B) PFS, and (C) OS in patients with or without bone therapy (n=110). (D) ORR, (E) PFS, and (F) OS in patients with or without bone-targeted therapy (n=110). (G) ORR, (H) $\mathrm{PFS}$, and (I) OS in patients with or without palliative bone radiotherapy $(\mathrm{n}=110) .{ }^{*}, \mathrm{P}<0.05$. ORR, objective response rate; PFS, progressionfree survival; OS, overall survival; PD, progressive disease; SD, stable disease; NR, not reached; PR, partial response.

targeted therapy. Although the OS was numerically longer, the difference was not statistically significant (NR $v$ s. 13.0 months, $\mathrm{P}=0.095)$. Bone-targeted therapy alone improved ORR (34.7\% vs. $18.4 \%, \mathrm{P}=0.005)$ and prolonged PFS (8.8 vs. 3.3 months, $\mathrm{P}=0.003$ ); however, palliative bone radiotherapy did not show significant response or survival benefits (ORR: $26.9 \%$ vs. 29.8\%, $\mathrm{P}=0.231$; PFS: 7.5 vs. 7.0 months, $\mathrm{P}=0.435$ ) (Figure 8 ).

According to treatment strategies, we found a significantly higher ORR when bone therapy was given with either first-line pembrolizumab monotherapy or combination (pembrolizumab monotherapy: $71.4 \%$ vs. $25.0 \%$; pembrolizumab combination therapy: $43.6 \%$ vs. $12.5 \%$, $\mathrm{P}<0.0001$ ); and PFS benefit only when it was added to firstline pembrolizumab combination therapy (PFS: $9.0 v s$. 1.8 months, $\mathrm{P}=0.009$ ) (Figure 9).

\section{Univariate and multivariate analyses}

The forest plots of the univariate analysis showed that histological subtype $(\mathrm{P}=0.012)$, liver metastases $(\mathrm{P}=0.043)$, bone therapy $(\mathrm{P}=0.002)$ and treatment line $(\mathrm{P}=0.004)$ had statistically significant correlations with $\mathrm{PFS}$ while ECOG PS score $(\mathrm{P}<0.0001)$, liver metastases $(\mathrm{P}=0.014)$ 


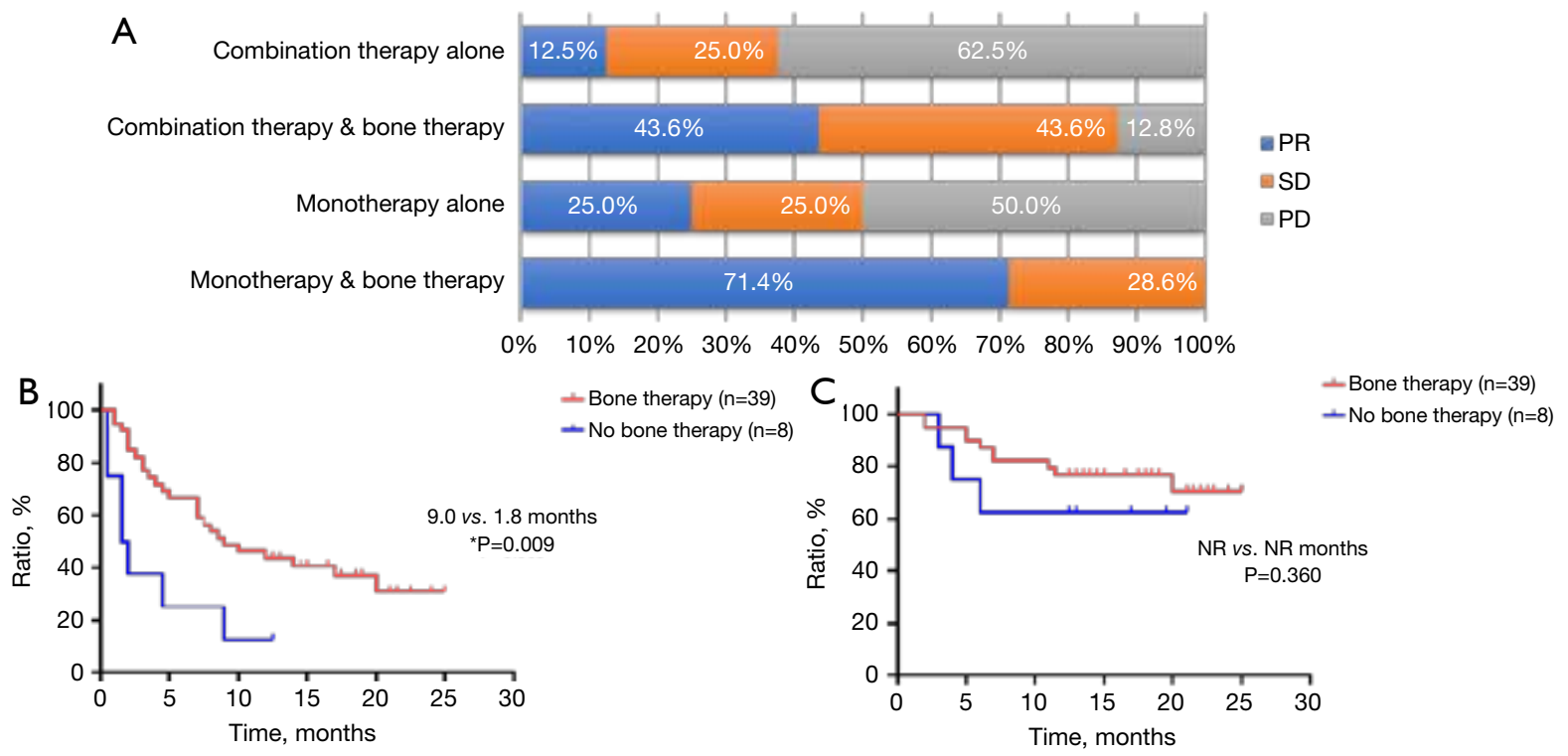

Figure 9 Efficacy and survival characteristics of patients receiving first-line immunotherapy and bone therapy. (A) Objective response rate, (B) progression-free survival, and (C) overall survival in patients receiving first-line pembrolizumab monotherapy or combination and bone therapy $(\mathrm{n}=110) .^{*}, \mathrm{P}<0.05$. PD, progressive disease; SD, stable disease; NR, not reached; PR, partial response.

and treatment line $(\mathrm{P}=0.001)$ resulted significant for $\mathrm{OS}$ (Figure 10). These factors were further included in a multivariate analysis. We found that lung adenocarcinoma $(\mathrm{HR}=0.343, \mathrm{P}=0.002)$ and bone therapy $(\mathrm{HR}=0.440$, $\mathrm{P}=0.001)$ were independent for PFS while ECOG PS score of $0-1(\mathrm{HR}=0.117, \mathrm{P}<0.0001)$ remained independent prognostic factors for OS. Besides, first-line pembrolizumab treatment $(\mathrm{PFS}$ : $\mathrm{HR}=0.620, \mathrm{P}=0.036$; OS: $\mathrm{HR}=0.372, \mathrm{P}=0.004)$ were independent for both $\mathrm{PFS}$ and OS (Table 2).

\section{Blood biomarkers}

Correlations between baseline serum levels of $\mathrm{LDH}$, NLR, and OS were evaluated. As shown in Figure 11, OS was negatively correlated with baseline serum levels of LDH and NLR (LDH: $r=0.345, P<0.0001$; NLR: $r=0.220$, $\mathrm{P}=0.021$ ). Furthermore, the optimal cut-off value of baseline serum levels of LDH and NLR were 240.5 IU/L and 5.55 , respectively. Survival analysis showed that the OS of patients with baseline serum LDH level $\leq 240.5$ IU/L (NR vs. 10.0 months, $\mathrm{P}<0.000$ ) or $\mathrm{NLR} \leq 5.55$ (NR vs. 18.0 months, $\mathrm{P}=0.039)$ was significantly extended compared with those with higher baseline serum levels of LDH or NLR than the cut-off value (Figure 12).

\section{Discussion}

At present, ICIs are widely used in advanced lung cancer, especially in NSCLC. The most common are PD-1/PD-L1 inhibitors. Compared to other ICIs, pembrolizumab is unique among the current immunotherapies because it has shown efficacy as a monotherapy when used as either second-line or first-line therapy for patients with advanced NSCLC (13). Based on significant survival benefits from KEYNOTE series clinical studies, pembrolizumab monotherapy has been approved as a first-line treatment for patients with $\mathrm{PD}-\mathrm{L} 1$ positive $(14,15)$, while firstline treatment combined with chemotherapy has become the standard treatment for patients with negative or low PD-L1 metastatic NSCLC (16). In KEYNOTE-189 clinical study, stage IV NSCLC patients with liver or brain metastases were selected, and subgroup analysis was performed. However, patients with bone metastases were not included. Until now, the efficacy of pembrolizumab therapy has not been fully confirmed. Probably lack of discussion on this population or the difficulty in measuring bone metastatic lesions and evaluating efficacy leads to little data. Therefore, our real-world retrospective study aimed to analyze the efficacy of immunotherapy, and demonstrated that pembrolizumab therapy could also be effective in NSCLC patients with bone metastases. Patients 


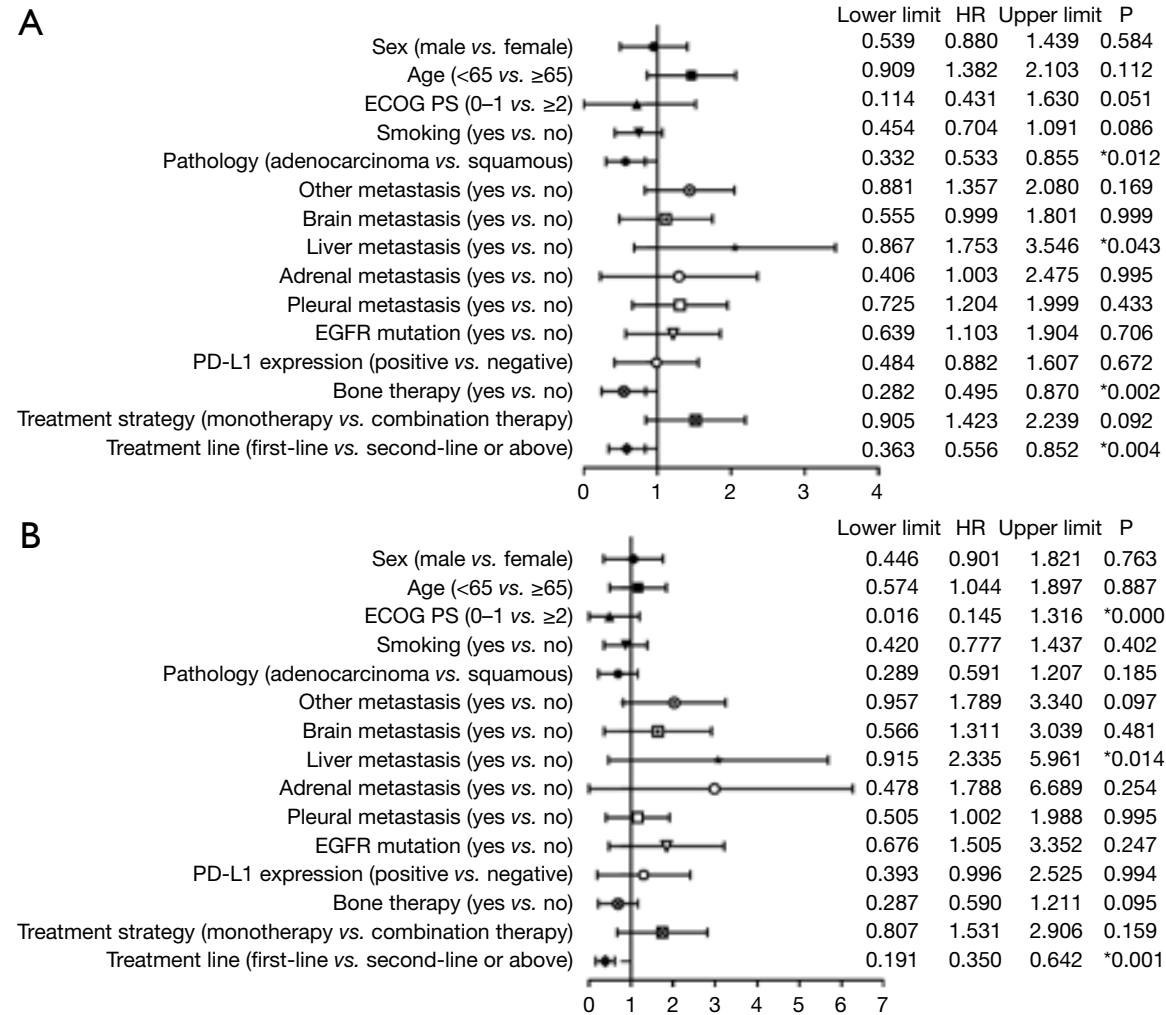

Figure 10 Forest plots for progression-free survival (A) and overall survival (B) for all patients. * $\mathrm{P}<0.05$. ECOG PS, Eastern Cooperation Oncology Group performance status; EGFR, epidermal growth factor receptor; OS, overall survival; PD-L1, programmed cell death-ligand 1; PFS, progression-free survival.

Table 2 Multivariate analyses of PFS and OS

\begin{tabular}{|c|c|c|c|c|c|c|c|c|}
\hline Subgroup & \multicolumn{4}{|c|}{ PFS } & \multicolumn{4}{|c|}{ OS } \\
\hline ECOG (0-1 vs. $\geq 2)$ & 0.457 & 0.179 & 1.166 & 0.101 & 0.117 & 0.040 & 0.345 & $<0.0001^{\star}$ \\
\hline $\begin{array}{l}\text { Pathology (adenocarcinoma vs. } \\
\text { squamous) }\end{array}$ & 0.343 & 0.172 & 0.686 & $0.002^{*}$ & 0.450 & 0.165 & 1.229 & 0.119 \\
\hline Liver metastasis (yes vs. no) & 1.251 & 0.682 & 2.293 & 0.469 & 1.236 & 0.545 & 2.806 & 0.612 \\
\hline Treatment line (1 vs. $\geq 2$ ) & 0.620 & 0.396 & 0.970 & $0.036^{*}$ & 0.372 & 0.190 & 0.728 & $0.004^{*}$ \\
\hline
\end{tabular}

*, P<0.05. PFS, progression-free survival; OS, overall survival; ECOG PS, Eastern Cooperation Oncology Group performance status; HR, hazard ratio.

who received palliative bone radiotherapy or bone-targeted therapy at the same time could particularly benefit from immunotherapy, and the significant effect was reflected on PFS by multivariate analysis.

Normal bone marrow plays a role in regulating the immune system and transporting different types of immune cells, including regulatory $\mathrm{T}$ cells, cytotoxic $\mathrm{T}$ cells, B cells, dendritic cells, and natural killer T cells (17). When NSCLC patients have bone metastases, the infiltrating tumor cells destroy the bones and produce PD-L1 and Chemokine (C-C motif) ligand 2 (CCL2), further promoting osteoclast differentiation and inducing 

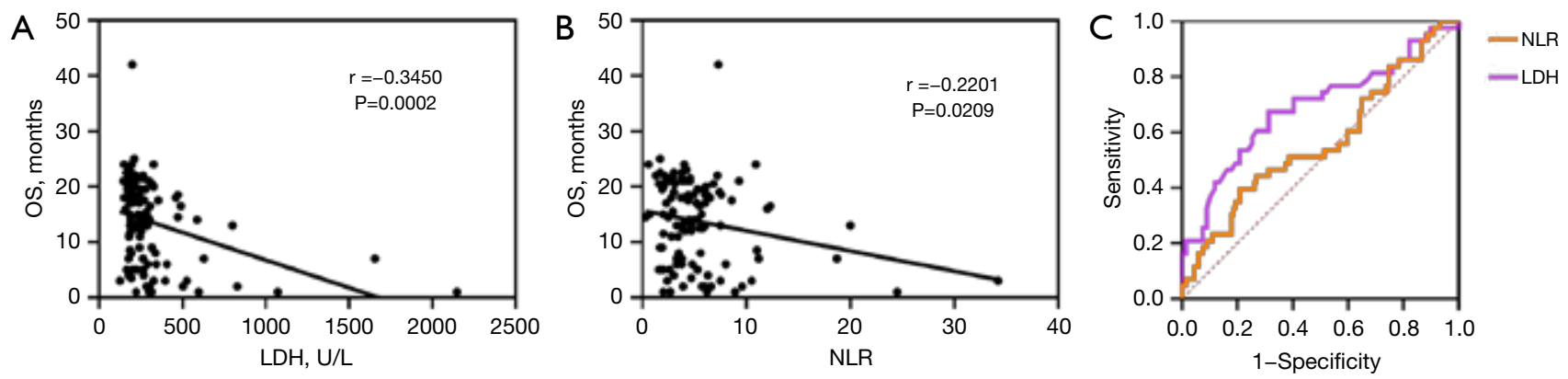

Figure 11 Blood biomarkers as independent predictors of OS. (A) Correlation of baseline LDH and OS. (B) Correlation of NLR and OS. (C) Receiver-operating characteristic curve $(\mathrm{n}=110)$. OS, overall survival; LDH, lactate dehydrogenase; NLR, neutrophil-to-lymphocyte ratio.
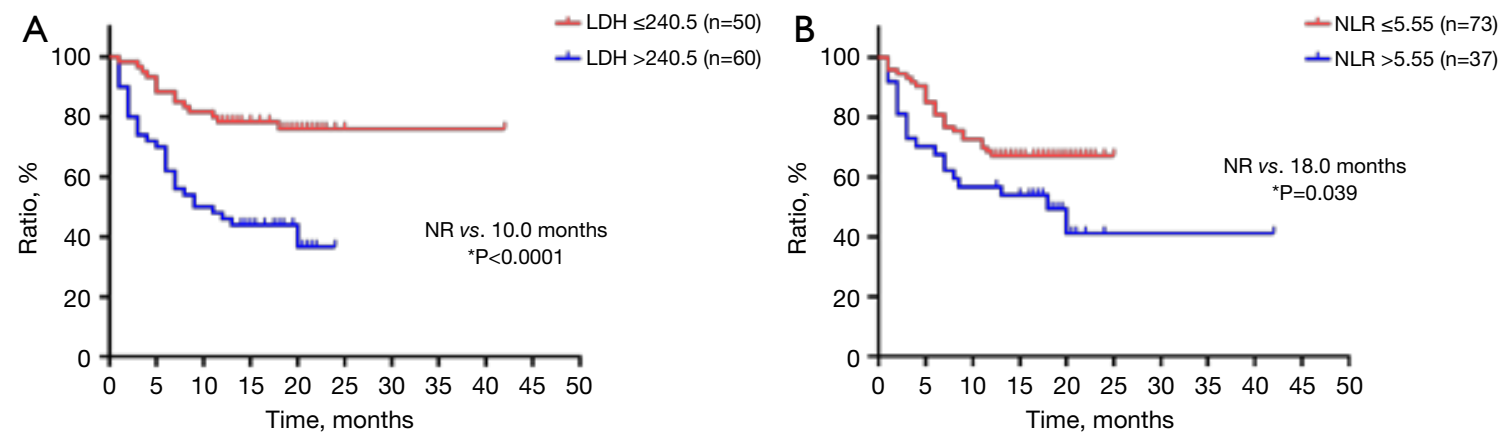

Figure 12 Overall survival of patients with cut-off values of baseline serum levels of (A) LDH and (B) NLR (n=110). *, P<0.05. NR, not reached; LDH, lactate dehydrogenase; NLR, neutrophil-to-lymphocyte ratio.

bone pain at the same time. Evidence has confirmed that the PD-1 monoclonal antibody can prevent the differentiation of osteoclast precursor cells to osteoclasts by inhibiting the production of CCL2, therefore improving the suppressive microenvironment (18). Actually, NSCLC patients with bone metastases in our study could benefit more from pembrolizumab therapy which is consistent with the literature reported.

By analyzing the treatment lines, we found that ORR of patients receiving first-line pembrolizumab therapy was significantly higher than those receiving second-line therapy or beyond. The benefits were also reflected in survival outcomes. Similarly, univariate and multivariate analyses showed that first-line immunotherapy was an independent predictor of PFS and OS. These results indicate that advanced NSCLC patients with bone metastases should receive immunotherapy as early as possible to improve the tumor microenvironment of the metastatic sites, therefore enhancing efficacy and prolonging survival. For patients receiving first-line immunotherapy, their initial immune status and low tumor heterogeneity enable better stimulation of immune activity to develop antitumor response.

The KEYNOTE-024 and KEYNOTE-042 studies indicated that advanced NSCLC patients with PD-L1 positive tumors could benefit from ICP monotherapy $(14,15)$. The results of KEYNOTE-189 showed that the median PFS and OS of first-line pembrolizumab combined with chemotherapy were 9.0 and 22.0 months, respectively, which nearly doubled compared with chemotherapy alone (median PFS: 4.9 months; median OS: 10.7 months) (16). The better efficacy of immunotherapy combined with chemotherapy may be that platinum-based drugs can induce immunogenic cell death, thereby recruiting dendritic cells to present antigens, and at the same time down-regulating PD-L1 expression of tumor cells to enhance T cell activity (19). A subgroup analysis of PD-L1 expression in patients treated with pembrolizumab combination therapy was performed in the KEYNOTE-189 study. The results showed that patients with PD-L1 $\geq 50 \%$ had more benefits in both efficacy and survival than other subgroups. Likewise, the subgroup of patients with detected PD-L1 expression in this retrospective study was analyzed. For patients whose 
PD-L1 expression $\geq 50 \%$, the ratio of $\mathrm{PR}$ after first-line combination therapy was significantly higher than that of monotherapy, confirming that combination therapy can reduce tumor load and relieve symptoms. In addition, we found that there was a statistical difference between pembrolizumab combination therapy and monotherapy in the PR proportion of patients with low and negative PD-L1. In brief, pembrolizumab could work as combination therapy when PD-L1 is low and negative, and could work better particularly for high PD-L1 NSCLC treated with first-line combination therapy.

Bone metastases were mainly distributed in the axial bone (20). Patients with only 1 bone metastatic site had a lighter tumor load; fewer bone-related events, including bone pain and pathological fractures; as well as a lower ECOG PS score. This resulted in longer survival compared with patients who had multiple bone metastases after receiving pembrolizumab therapy. Bone therapy included palliative bone radiotherapy and bone-targeted therapy. However, patients who underwent local bone surgery were excluded from our study because their ECOG PS score was $>2$ and they did not have the opportunity to receive immunotherapy. Our results showed that immunotherapy and bone therapy contributed significantly to disease remission. Bone-targeted therapy, based on bisphosphonates, is also beneficial to control disease through inhibiting the activity of osteoclasts and relieving symptoms. Bone radiotherapy can induce apoptosis of tumor cells directly to release neoantigens and activate immune response. In addition, it can ease pain locally and is often used as a palliative treatment for bone metastases. There have been a number of studies demonstrating the synergistic effect of immunotherapy combined with palliative bone radiotherapy (21). In the present study, the synergistic effect was not seen, mainly because some patients did not meet the indications for radiotherapy. Others received palliative bone radiotherapy of only 1-2 lesions to relieve pain as a result of too many metastatic lesions. Systemic immunotherapy combined with palliative bone radiotherapy or bone-targeted therapy could be used for disease control and improving survival for advanced NSCLC patients with bone metastases.

The standard first-line treatment for advanced NSCLC patients who are EGFR mutation positive is EGFR-tyrosine kinase inhibitor (TKI) targeted therapy. Generally speaking, EGFR mutation positivity is considered to be a negative factor for immunotherapy. However, among patients with bone metastases included in our study, we could not detect any significant survival differences with pembrolizumab based treatment according to the EGFR status.

The ECOG PS score reflects the physical status of advanced NSCLC patients. The lower the score, the higher the acceptance of immunotherapy and the better the prognosis. Univariate and multivariate analyses showed that the OS of patients with ECOG PS score of $0-1$ significantly improved after immunotherapy, and was therefore considered an independent predictor of OS. Adenocarcinoma is the most common type of NSCLC and has the highest incidence of bone metastases (22). For adenocarcinoma patients, immunotherapy could significantly delay disease progression. The bone, liver, brain, adrenal gland, and pleura were common sites of metastases in advanced NSCLC patients (23). Distant metastasis is also the main cause of cancer-related death (24). More metastatic sites are associated with a worse prognosis. In the present study, accompanying liver metastases aggravated the metastatic burden and shortened survival. Early immunotherapy should be used for patients with low metastatic burden to enhance antitumor response and promote therapeutic outcomes.

White blood cells, neutrophil and lymphocyte count, and serum levels of LDH and NLR are considered potential inflammatory biomarkers. Under aerobic conditions, tumor cells consume high levels of glucose to meet the needs of rapid proliferation, resulting in an increase in lactic acid production. This process is catalyzed by LDH (25). Elevated serum LDH level is associated with poor prognosis of lung cancer (26). Inflammation, which stimulates the production and release of neutrophils and simultaneously decreases the production of lymphocytes, is common in patients with bone metastases (27). As reported in the literature, NLR in combination with PD-L1 or LDH have been regarded as biomarkers for high PD-L1 NSCLC treated with first-line pembrolizumab (28). In this retrospective study, we found a significant negative correlation between OS and baseline serum levels of LDH or NLR. Baseline serum LDH >240.5 IU/L or NLR $>5.55$ was associated with shorter OS, which indicated that baseline serum LDH and NLR could be prognostic factors of bone metastases. In addition, analyzed combined baseline serum levels of LDH with NLR, significant predictive value was not seen (data not shown).

The present study has several limitations. First, we could not assess the response of bone lesions and the evaluation was performed only for the measurable target lesion, not bone metastatic sites. Bone metastases are an exception 
in response assessment guidelines, because of the nature of the fixed bony defects, their complexity, which ranges from sclerotic to osteolytic. Second, this was a real-world retrospective study and some clinical data were missing or incomplete. Third, the PD-L1 expression of half the patients was unknown. The technology and platform of PD-L1 detection have not been popularized in major hospitals in China, especially before 2019. This led to the missing detection results and an incomprehensive analysis of PD-L1 expression. Finally, because few patients who received palliative bone radiotherapy were enrolled in the study, the correlation between radiotherapy doses, sites, and treatment efficacy was not discussed in details. Therefore, studies with larger sample sizes are needed for further analysis.

\section{Conclusions}

The findings of our retrospective study indicate that pembrolizumab therapy is effective in advanced NSCLC patients with bone metastases, and bone therapy (including palliative bone radiotherapy and bone-targeted therapy) may be beneficial. Baseline serum LDH level $\leq 240.5$ IU/L and NLR $\leq 5.55$ could predict their prognosis following immunotherapy.

\section{Acknowledgments}

The authors appreciate the academic support from the AME Lung Cancer Collaborative Group.

Funding: This study was funded by the Western Medicine Guide Project of Shanghai Committee of Science and Technology (grant No. 18411968500).

\section{Footnote}

Reporting Checklist: The authors have completed the STROBE reporting checklist. Available at https://tlcr. amegroups.com/article/view/10.21037/tlcr-21-1033/rc

Data Sharing Statement: Available at https://tlcr.amegroups. com/article/view/10.21037/tlcr-21-1033/dss

Conflicts of Interest: All authors have completed the ICMJE uniform disclosure form (available at https://tlcr.amegroups. com/article/view/10.21037/tlcr-21-1033/coif). Dr. AA reports that he received personal fees for attending advisory from Bristol-MyersSquibb, AstraZeneca, Roche, Pfizer, Merck Sharp and Dohme, Astella, Eli Lilly and Boehringer-
Ingelheim and received fees for speaking bureau for Eli Lilly, AstraZeneca, MSD for work performed outside of the current study. Dr. GLB has received consulting fees from AstraZeneca, and payment or honoraria for lectures, presentations, speakers' bureaus, manuscript writing or educational events from AstraZeneca and Astellas. Dr. IJO has received research funding from Roche, and consulting fees from Roche, Ono, MSD, Pfizer, Boehringer-Ingelheim, AstraZeneca and Takeda. The other authors have no conflicts of interest to declare.

Ethical Statement: The authors are accountable for all aspects of the work in ensuring that questions related to the accuracy or integrity of any part of the work are appropriately investigated and resolved. All procedures performed in this study involving human participants were in accordance with the Declaration of Helsinki (as revised in 2013). The study was approved by the Ethics Committee of Shanghai Chest Hospital Affiliated to Shanghai Jiao Tong University (No. IS21110) and informed consent was taken from all the patients.

Open Access Statement: This is an Open Access article distributed in accordance with the Creative Commons Attribution-NonCommercial-NoDerivs 4.0 International License (CC BY-NC-ND 4.0), which permits the noncommercial replication and distribution of the article with the strict proviso that no changes or edits are made and the original work is properly cited (including links to both the formal publication through the relevant DOI and the license). See: https://creativecommons.org/licenses/by-nc-nd/4.0/.

\section{References}

1. Sung H, Ferlay J, Siegel RL, et al. Global Cancer Statistics 2020: GLOBOCAN Estimates of Incidence and Mortality Worldwide for 36 Cancers in 185 Countries. CA Cancer J Clin 2021;71:209-49.

2. Doroshow DB, Sanmamed MF, Hastings K, et al. Immunotherapy in Non-Small Cell Lung Cancer: Facts and Hopes. Clin Cancer Res 2019;25:4592-602.

3. Pasello G, Pavan A, Attili I, et al. Real world data in the era of Immune Checkpoint Inhibitors (ICIs): Increasing evidence and future applications in lung cancer. Cancer Treat Rev 2020;87:102031.

4. Miller KD, Nogueira L, Mariotto AB, et al. Cancer treatment and survivorship statistics, 2019. CA Cancer J Clin 2019;69:363-85.

5. Kuchuk M, Addison CL, Clemons M, et al. Incidence and 
consequences of bone metastases in lung cancer patients. J Bone Oncol 2013;2:22-9.

6. Coleman RE. Metastatic bone disease: clinical features, pathophysiology and treatment strategies. Cancer Treat Rev 2001;27:165-76.

7. Janjan N, Lutz ST, Bedwinek JM, et al. Therapeutic guidelines for the treatment of bone metastasis: a report from the American College of Radiology Appropriateness Criteria Expert Panel on Radiation Oncology. J Palliat Med 2009;12:417-26.

8. LeVasseur N, Clemons M, Hutton B, et al. Bone-targeted therapy use in patients with bone metastases from lung cancer: A systematic review of randomized controlled trials. Cancer Treat Rev 2016;50:183-93.

9. Myoken Y, Fujita Y, Kawamoto K, et al. Osteonecrosis of the jaw in a metastatic lung cancer patient with bone metastases undergoing pembrolizumab + denosumab combination therapy: Case report and literature review. Oral Oncol 2020;111:104874.

10. Mezquita L, Auclin E, Ferrara R, et al. Association of the Lung Immune Prognostic Index With Immune Checkpoint Inhibitor Outcomes in Patients With Advanced Non-Small Cell Lung Cancer. JAMA Oncol 2018;4:351-7.

11. Tavakkoli M, Wilkins CR, Mones JV, et al. A Novel Paradigm Between Leukocytosis, G-CSF Secretion, Neutrophil-to-Lymphocyte Ratio, Myeloid-Derived Suppressor Cells, and Prognosis in Non-small Cell Lung Cancer. Front Oncol 2019;9:295.

12. Eisenhauer EA, Therasse P, Bogaerts J, et al. New response evaluation criteria in solid tumours: revised RECIST guideline (version 1.1). Eur J Cancer 2009;45:228-47.

13. Peters S, Kerr KM, Stahel R. PD-1 blockade in advanced NSCLC: A focus on pembrolizumab. Cancer Treat Rev 2018;62:39-49.

14. Reck M, Rodríguez-Abreu D, Robinson AG, et al. Updated Analysis of KEYNOTE-024: Pembrolizumab Versus Platinum-Based Chemotherapy for Advanced NonSmall-Cell Lung Cancer With PD-L1 Tumor Proportion Score of 50\% or Greater. J Clin Oncol 2019;37:537-46.

15. Mok TSK, Wu YL, Kudaba I, et al. Pembrolizumab versus chemotherapy for previously untreated, PD-L1expressing, locally advanced or metastatic non-small-cell lung cancer (KEYNOTE-042): a randomised, open-label, controlled, phase 3 trial. Lancet 2019;393:1819-30.

16. Gadgeel S, Rodríguez-Abreu D, Speranza G, et al. Updated Analysis From KEYNOTE-189: Pembrolizumab or Placebo Plus Pemetrexed and Platinum for Previously Untreated Metastatic Nonsquamous Non-Small-Cell
Lung Cancer. J Clin Oncol 2020;38:1505-17.

17. Landi L, D'Incà F, Gelibter A, et al. Bone metastases and immunotherapy in patients with advanced non-small-cell lung cancer. J Immunother Cancer 2019;7:316.

18. Wang K, Gu Y, Liao Y, et al. PD-1 blockade inhibits osteoclast formation and murine bone cancer pain. J Clin Invest 2020;130:3603-20.

19. Hato SV, Khong A, de Vries IJ, et al. Molecular pathways: the immunogenic effects of platinum-based chemotherapeutics. Clin Cancer Res 2014;20:2831-7.

20. Krishnamurthy GT, Tubis M, Hiss J, et al. Distribution pattern of metastatic bone disease. A need for total body skeletal image. JAMA 1977;237:2504-6.

21. Selvaggi G, Scagliotti GV. Management of bone metastases in cancer: a review. Crit Rev Oncol Hematol 2005;56:365-78.

22. Sugiura H, Yamada K, Sugiura T, et al. Predictors of survival in patients with bone metastasis of lung cancer. Clin Orthop Relat Res 2008;466:729-36.

23. Hess KR, Varadhachary GR, Taylor SH, et al. Metastatic patterns in adenocarcinoma. Cancer 2006;106:1624-33.

24. Turajlic S, Swanton C. Metastasis as an evolutionary process. Science 2016;352:169-75.

25. Hsu PP, Sabatini DM. Cancer cell metabolism: Warburg and beyond. Cell 2008;134:703-7.

26. Giroux Leprieur E, Lavole A, Ruppert AM, et al. Factors associated with long-term survival of patients with advanced non-small cell lung cancer. Respirology 2012;17:134-42.

27. Thio QCBS, Goudriaan WA, Janssen SJ, et al. Prognostic role of neutrophil-to-lymphocyte ratio and platelet-tolymphocyte ratio in patients with bone metastases. Br J Cancer 2018;119:737-43.

28. Banna GL, Signorelli D, Metro G, et al. Neutrophil-tolymphocyte ratio in combination with $\mathrm{PD}-\mathrm{L} 1$ or lactate dehydrogenase as biomarkers for high PD-L1 non-small cell lung cancer treated with first-line pembrolizumab. Transl Lung Cancer Res 2020;9:1533-42.

(English Language Editor: R. Soctt)

Cite this article as: Qiang $\mathrm{H}$, Lei $\mathrm{Y}$, Shen $\mathrm{Y}$, Li J, Zhong $\mathrm{H}$ Zhong R, Zhang X, Chang Q, Lu J, Feng H, Zhu Y, Addeo A, Banna GL, Oh IJ, Qian J, Chu T. Pembrolizumab monotherapy or combination therapy for bone metastases in advanced nonsmall cell lung cancer: a real-world retrospective study. Transl Lung Cancer Res 2022;11(1):87-99. doi: 10.21037/tlcr-211033 